\title{
RISCO CARDIOVASCULAR DE USUÁRIOS DE UM CENTRO DE ATENÇÃO PSICOSSOCIAL EM ÁLCOOL E DROGAS
}

\section{Cardiovascular risk in users of an alcohol and drug psychosocial care center \\ Riesgo cardiovascular en usuarios de un centro de atención psicosocial de alcohol y droga}

\author{
Kamylla Karolynne Bezerra Pontes (iD \\ Universidade Ceuma - UNICEUMA - São Luís (MA) - Brasil \\ Ester Barbosa Soares (iD \\ Universidade Ceuma - UNICEUMA - São Luís (MA) - Brasil
}

Alexsandro Ferreira dos Santos (iD

Universidade Ceuma - UNICEUMA - São Luís (MA) - Brasil

Adrielle Zagmignan (iD

Universidade Ceuma - UNICEUMA - São Luís (MA) - Brasil

Eliziane Gomes da Costa Moura da Silva (iD

Universidade Ceuma - UNICEUMA - São Luís (MA) - Brasil

Izabela Correa Costa iD

Universidade Ceuma - UNICEUMA - São Luís (MA) - Brasil

Virgínia Nunes Lima ii

Universidade Ceuma - UNICEUMA - São Luís (MA) - Brasil

Janaina Maiana Abreu Barbosa (iD)

Universidade Ceuma - UNICEUMA - São Luís (MA) - Brasil

\section{RESUMO}

Objetivo: Avaliar o risco cardiovascular de usuários de um Centro de Atenção Psicossocial em Álcool e Drogas. Métodos: Estudo retrospectivo e analítico, realizado em um Centro de Atenção Psicossocial em Álcool e Drogas em São Luís, Maranhão. A amostra compôs-se por prontuários de homens adultos atendidos nos anos de 2015 a 2017. Os prontuários de usuários exclusivos de álcool não foram incluídos no estudo. Coletaram-se informações referentes aos aspectos socioeconômicos, demográficos, estilo de vida, histórico de dependência química, além das informações antropométricas. Utilizou-se o programa Stata ${ }^{\circledR}$, versão 13.0, para análise dos dados, sendo realizada regressão de Poisson. Resultados: Dos 122 prontuários analisados, 73,5\% ( $\mathrm{n}=89$ ) pertenciam à faixa etária de 20 a 39 anos, $79,5 \%(n=97)$ eram solteiros e 49,2\% ( $n=60)$ possuíam ensino fundamental incompleto ou completo. Quanto ao estado nutricional, 54,9\% $(n=67)$ dos dependentes estavam eutróficos, 18,0\% $(n=22)$ deles estavam em risco para doenças cardiovasculares, de acordo com a circunferência da cintura, e também 17,2 \% ( $n=21$ ) pela razão cintura/ estatura. Além disso, 36,6\% ( $n=41)$ e 41,8\% $(n=51)$ dos dependentes permaneceram com risco de doença cardiovascular conforme a relação cintura/quadril e o índice de conicidade, respectivamente. Apresentar idade entre 40 e 59 anos (OR = 4,40; IC 95\%: 1,52-12,75) e utilizar cocaína (OR = 3,27; IC 95\%: 1,15 - 9,27) foram fatores de risco para doenças cardiovasculares pela razão cintura/estatura. Conclusão: $O$ estudo identificou como dependentes químicos adultos jovens, eutróficos, com risco de doença cardiovascular e associação significativa da razão cintura/estatura com a idade e a utilização de cocaína.

Descritores: Usuário de Drogas; Drogas llícitas; Fatores de Risco.

\section{ABSTRACT}

Objective: To assess cardiovascular risk in users of an Alcohol and Drugs Psychosocial Care Center. Methods: A retrospective and analytical study was carried out at an Alcohol and Drugs Psychosocial Care Center in São Luís, Maranhão. The sample consisted of medical records of adult men served from 2015 to 2017. The medical records of individuals who used only alcohol

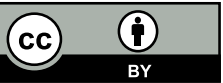


were not included in the study. Socioeconomic, demographic, lifestyle, history of chemical dependence, and anthropometric information were collected. The data were analyzed in Stata ${ }^{\circledR}$ version 13.0 using Poisson regression. Results: Of the 122 medical records analyzed, $73.5 \%(n=89)$ were aged 20 to 39 years, $79.5 \%(n=97)$ were single and $49.2 \%(n=60)$ had either incomplete or complete primary education. Regarding nutritional status, $54.9 \%(n=67)$ of the individuals presented normal values and $18.0 \%$ $(n=22)$ were at risk for cardiovascular diseases based on waist circumference and $17.2 \%(n=21)$ were at cardiovascular risk based on waist-to-height ratio. In addition, $36.6 \%(n=41)$ and $41.8 \%(n=51)$ of the individuals remained at risk of cardiovascular disease based on waist-to-hip ratio and conicity index, respectively. Being aged 40-59 years (OR $=4.40,95 \% \mathrm{Cl}$ : 1.52-12.75) and using cocaine (OR = 3.27, 95\% Cl: 1.15 - 9.27) were risk factors for cardiovascular diseases based on waist-to-height ratio. Conclusion: The study identified that chemically dependents were young adults at normal weight at risk for cardiovascular disease and waist-to-height ratio was significantly associated with age and cocaine use.

Descriptors: Drug Users; Illicit Drugs; Risk Factors.

\section{RESUMEN}

Objetivo: Evaluar el riesgo cardiovascular de usuarios de un Centro de Atención Psicosocial de Alcohol y Droga. Métodos: Estudio retrospectivo y analítico realizado en un Centro de Atención Psicosocial de Alcohol y Droga de São Luís, Maranhão. La muestra ha sido identificada de historiales clínicos de hombres adultos asistidos entre los años 2015 y 2017. Los historiales de usuarios exclusivos de alcohol no han sido incluidos en el estudio. Se recogieron informaciones de los aspectos socioeconómicos, los demográficos, del estilo de vida, del histórico de dependencia química además de las informaciones antropométricas. Se utilizó el programa Stata ${ }^{\circledR}$ versión 13.0 y para el análisis de los datos ha sido realizada la regresión de Poisson. Resultados: De los 122 historiales analizados, el 73,5\% (n=89) estaban en la franja de edad entre 20 y 39 años, el 79,5\% (n=97) eran solteros y el 49,2\% ( $n=60)$ tenían la educación primaria incompleta o completa. Sobre el estado nutricional, el $54,9 \%$ ( $n=67)$ de los dependientes eran eutróficos, el 18,0\% $(n=22)$ de ellos tenían riesgo para enfermedades cardiovasculares según la circunferencia de la cintura y también el 17,2 \% (n=21) por la razón cintura/estatura. Además de eso, el 36,6\% ( $n=41)$ y el 41,8\% (n=51) de los dependientes permanecieron con el riesgo para enfermedad cardiovascular según la relación cintura/cuadril y el índice de conicidad, respectivamente. Tener la edad entre 40 y 59 años (OR = 4,40; IC 95\%: 1,52-12,75) y utilizar cocaína (OR = 3,27; IC 95\%: 1,15 - 9,27) han sido los factores de riesgo para enfermedades cardiovasculares por la razón cintura/estatura. Conclusión: El estudio ha identificado los adultos jóvenes, eutróficos y con riesgo de enfermedad cardiovascular como dependientes químicos y asociación significativa de la razón cintura/estatura con la edad y la utilización de cocaína.

Descriptores: Consumidores de Drogas; Drogas llícitas; Factores de Riesgo.

\section{INTRODUÇÃO}

A dependência química é considerada uma patologia crônica, capaz de alterar as funções orgânicas do indivíduo, levando a mudanças físicas e comportamentais ${ }^{(1)}$. O aumento do consumo de drogas é preocupante pela quantidade de dependentes químicos que vem se formando, tornando-se um caso de saúde pública(2).

De acordo com o relatório mundial das Nações Unidas sobre Drogas e Crime (UNODC), cerca de 247 milhões de indivíduos no mundo, com idade de 15 a 64 anos, já utilizaram algum tipo de substância ilícita, sendo mais frequente entre homens, que têm preferência por maconha, cocaína e anfetaminas ${ }^{(3)}$. No Brasil, a pesquisa do II Levantamento Nacional de Álcool e Drogas (LENAD) estimou que o consumo da substância mais prevalente entre a população adulta é a maconha $(5,8 \%)$, seguida de cocaína $(3,8 \%)$, estimulantes $(2,7 \%)$ e $\operatorname{crack}(0,7 \%)^{(4)}$.

Para acompanhar os dependentes químicos, o Ministério da Saúde, através da Política Nacional de Saúde Mental, Álcool e Outras Drogas, possibilita a implantação de centros especializados no tratamento de usuários de álcool e outras drogas. A referência para o atendimento é a Rede de Atenção Psicossocial, que promove ações de redução de danos em conjunto com as redes de saúde, como Unidades Básicas de Saúde, Centros de Atenção Psicossocial, Prontos-socorros e outros ${ }^{(5)}$.

O Centro de Atenção Psicossocial em Álcool e Drogas (CAPS AD) atende pessoas de todas as faixas etárias, em sofrimento psíquico decorrente de crack, álcool e outras drogas, nos municípios ou regiões que possuem mais de cento e cinquenta mil habitantes ${ }^{(6)}$. Oferta atendimento estratégico e abrangente através do Sistema Único de Saúde (SUS), e conta com uma equipe multidisciplinar, na qual os pacientes assistidos participam de reuniões em grupos, atendimento individual, consultas médicas e oficinas terapêuticas ${ }^{(5)}$.

A utilização de drogas psicoativas acarreta distúrbios, causados pela ação da substância e de fatores hereditários, ambientais e compulsórios, sofrendo alteração na ação conforme o tipo e a faixa etária em que se encontram os indivíduos $^{(7)}$. Essas substâncias alteram o sistema nervoso central (SNC), tornando-os susceptíveis a doenças, principalmente àquelas relacionadas ao sistema cardiovascular ${ }^{(8)}$. A cocaína está associada a diversas doenças 
cardiovasculares (DCV), como hipertensão, infarto do miocárdio, taquiarritmias, dissecção da aorta, insuficiência cardíaca, vasoespasmo e aterogênese acelerada, decorrente do infarto( ${ }^{(9)}$.

O uso abusivo de cocaína é uma das principais demandas pelo serviço público de saúde relacionado aos sintomas cardiovasculares ${ }^{(10)}$. Entre os atendimentos referentes ao consumo de cocaína, $39,5 \%$ dos indivíduos queixam-se de dor torácica aguda e infarto agudo do miocárdio. Destes, cerca de 2 a $7 \%$ são por dor torácica aguda causada por uso de substâncias ilícitas, sendo a cocaína responsável por $25 \%$ das causas de infarto entre pessoas de 18 a 45 anos $^{(8)}$.

Devido à exposição dos dependentes químicos à doença cardíaca, torna-se de fundamental importância para a promoção da saúde avaliar os fatores de risco cardiovascular desse público. Ao considerar os prejuízos provocados pelo vício, o objetivo do estudo foi avaliar o risco cardiovascular de usuários de um Centro de Atenção Psicossocial em Álcool e Drogas.

\section{MÉTODOS}

Estudo retrospectivo e analítico, realizado em um CAPS AD localizado na cidade de São Luís, Maranhão, Brasil, que atende pessoas com diferentes faixas etárias, com transtornos psíquicos decorrentes da utilização de crack, álcool e outras drogas. Realizou-se a coleta de dados no período de março e abril de 2018, com prontuários de pacientes em tratamento para dependência química atendidos nos anos de 2015 a 2017.

A amostragem, do tipo não probabilística, incluía 122 prontuários de homens adultos. Os prontuários de usuários exclusivos de álcool não compuseram o estudo, pois o enfoque da pesquisa eram usuários de drogas psicoativas ilícitas.

O formulário utilizado na coleta de dados continha perguntas de aspectos socioeconômicos, demográficos, estilo de vida, histórico de dependência química, tipo de droga utilizada, idade de início do uso, período do tratamento, motivo de internação e tipo de tratamento. Além dos dados antropométricos, como peso e altura, para cálculo do Índice de Massa Corporal (IMC), foram coletados nos prontuários a circunferência da cintura (CC) e a circunferência do quadril (CQ).

As variáveis demográficas, socioeconômicas e de estilo de vida foram categorizadas em idade (20 a 39 anos e 40 a 59 anos), estado civil (vive com companheiro ou sem companheiro), escolaridade (ensino fundamental incompleto ou completo, ensino médio incompleto ou completo, e superior incompleto ou pós-graduação) e prática de atividade física (sim ou não).

Em relação às variáveis de histórico de dependência química, consideraram-se os seguintes aspectos: as substâncias que os usuários já fizeram uso (álcool, tabaco, cola, maconha, solvente inalantes, loló, cocaína, crack e merla), a idade de início do uso ( $<12$ anos, 12 - 15 anos, 15 - 18 anos, 18 - 21 anos, $>21$ anos), o período de tratamento (até 1 mês, de 1 a 6 meses, > 6meses) e o motivo de internação (dependência por álcool e drogas e dependência por drogas).

Para classificar o IMC, aplicaram-se os pontos de corte preconizados pela Organização Mundial da Saúde ${ }^{(11)}$. Segundo a Associação Brasileira para o Estudo da Obesidade e da Síndrome Metabólica ${ }^{(12)}$, os homens com $C C \geq 94 \mathrm{~cm}$ expressaram risco aumentado de $\mathrm{DCV}$ e, $\mathrm{com} \geq 102 \mathrm{~cm}$, risco muito aumentado para DCV.

A relação cintura/estatura (RCEst) é indicativo de risco cardiovascular quando o valor for igual ou superior a 0,52 . O índice de conicidade (IC) foi obtido por meio da relação da $\mathrm{CC}(\mathrm{cm}) /[0,109 \times$ (peso corporal $(\mathrm{kg}) /$ estatura $(\mathrm{m})$ ], adotada como ponto de corte $\geq 1,25^{(13)}$. A razão cintura/quadril $(R C Q)$ foi obtida a partir da relação entre $\mathrm{CC}(\mathrm{cm})$ e $\mathrm{CQ}(\mathrm{cm})$ e considerado elevado os valores maiores que 0,95 para homens ${ }^{(14)}$.

Realizou-se a análise e tabulação dos dados no programa Stata®, versão 13.0. Descreveu-se a análise das variáveis qualitativas por frequência e a análise multivariada, pelo método de regressão de Poisson, no qual se incluiu em modelo múltiplo todas as variáveis associadas ao evento de interesse com significância estatística de até $20 \%$. Para aceitação das associações investigadas no modelo final, adotou-se o valor de $p<0,05$. Considerou-se como variável desfecho a RCEst, sendo as variáveis independentes a situação socioeconômica e demográfica e o histórico de uso de drogas.

Esta pesquisa recebeu aprovação do Comitê de Ética em Pesquisa (CEP) da Universidade Ceuma (UNICEUMA), sob o Parecer n. ${ }^{\circ} 2.519 .436$.

\section{RESULTADOS}

Analisaram-se 122 prontuários de dependentes químicos, sendo que $73 \%(n=89)$ pertenciam à faixa etária de 20 a 39 anos, $79,5 \%(n=97)$ deles viviam sem companheira e 49,2\% ( $n=60)$ possuíam ensino fundamental completo ou 
incompleto. Sobre o estilo de vida, 62,3\% ( $\mathrm{n}=76)$ dos dependentes químicos praticavam atividade física (Tabela I).

Tabela I - Características socioeconômicas, demográficas e estilo de vida de usuários de um Centro de Atenção Psicossocial em Álcool e Drogas. São Luís, Maranhão, 2018.

\begin{tabular}{lcc}
\hline Variáveis & $\mathbf{n}$ & $\%$ \\
\hline Idade (em anos) & 89 & 73 \\
$20-39$ & 33 & 27 \\
$\quad 40-59$ & & \\
Estado civil & 97 & 79,5 \\
$\quad$ Sem companheira & 25 & 20,5 \\
$\quad$ Com companheira & & \\
Escolaridade & 60 & 49,2 \\
$\quad$ Ensino fundamental completo & & \\
Ensino fundamental incompleto & 52 & 42,6 \\
$\quad$ Ensino médio completo & & 8,2 \\
$\quad$ Ensino médio incompleto & 10 & \\
Ensino superior completo & & 62,3 \\
Ensino superior incompleto & & 37,7 \\
Atividade física & 76 & 100 \\
$\quad$ Sim & 46 & \\
$\quad$ Não & 122 & \\
Total & & \\
\hline
\end{tabular}

Quanto ao histórico de dependência química, observou-se que 63,1\% ( $n=77)$ eram dependentes tanto de álcool quanto de drogas, $62,3 \%(n=76)$ frequentaram o CAPS AD em um período de 1 a 6 meses para tratamento e $64 \%$ $(n=78)$ dos dependentes iniciaram o consumo de drogas com idade de entre 12 e 18 anos (Tabela II).

Tabela II - Histórico de dependência química de usuários assistidos em um Centro de Atenção Psicossocial em Álcool e Drogas. São Luís, Maranhão, 2018.

\begin{tabular}{lcc}
\hline Variáveis & $\mathbf{n}$ & $\%$ \\
\hline Motivo da internação & & \\
$\quad$ Dependência por Álcool e Droga & 77 & 63,1 \\
$\quad$ Dependência por drogas & 45 & 36,9 \\
Período do tratamento & 5 & 4,1 \\
Até 1 mês & 76 & 62,3 \\
1 - 6 meses & 41 & 33,6 \\
$>6$ meses & & \\
Idade que iniciou o uso de droga & 15 & 12,3 \\
$<12$ anos & 78 & 64 \\
$12-18$ anos & 29 & 23,7 \\
$>18$ anos & 122 & 100 \\
\hline
\end{tabular}

No que se refere às substâncias que os pacientes já fizeram uso, o crack foi a substância psicoativa mais utilizada $(84,3 \%, n=102)$, seguida por maconha $(80,2 \%, n=97)$, álcool $(77,7 \%, n=94)$, merla $(48,8 \%, n=59)$, cocaína $(40,5 \%, n=49)$, tabaco $(28,1 \%, n=34)$, cola/solvente $(5,0 \%, n=6)$ e loló $(2,5 \%, n=3)$.

A Tabela III mostra as características antropométricas dos participantes. De acordo com o IMC, 54,9\% ( $n=67$ ) dos indivíduos estavam eutróficos. No entanto, com relação a CC e a RCEst, 18,0\% ( $n=22)$ e 17,2\% $(n=21)$ estavam com risco para DCV, respectivamente. Conforme a RCQ, 33,6\% $(n=41)$ apresentaram risco para DCV e, quanto ao IC, $41,8 \%(n=51)$ também se mantiveram com risco de desenvolver DCV. 
Tabela III - Características antropométricas de usuários assistidos em um Centro de Atenção Psicossocial em Álcool e Drogas. São Luís, Maranhão, 2018.

\begin{tabular}{lcc}
\hline Variáveis & $\mathbf{n}$ & \% \\
\hline IMC & 67 & 54,9 \\
Eutrofia & 37 & 30,3 \\
Sobrepeso & 18 & 14,8 \\
Obesidade & & \\
CC & 100 & 82,0 \\
$\quad$ Sem risco de DCV & 22 & 18,0 \\
$\quad$ Com risco de DCV & & \\
RCEst & 101 & 82,8 \\
Sem risco de DCV & 21 & 17,2 \\
Com risco de DCV & & 66,4 \\
RCQ & 81 & 33,6 \\
Sem risco DCV & 41 & \\
Com risco de DCV & & 58,2 \\
IC & 71 & 41,8 \\
Sem risco DCV & 51 & 100 \\
$\quad$ Com risco de DCV & 122 & \\
Total
\end{tabular}

IMC: índice de massa corporal; CC: circunferência da cintura; RCEst: razão circunferência cintura/estatura; RCQ: relação cintura/quadril; IC: índice de conicidade; DCV: doenças cardiovasculares

Apresentar idade entre 40 e 59 anos (OR de 4,13; IC 95\%: 1,54 - 11,05), estado civil com companheira (OR de 2,30; IC 95\%: 0,81 - 6,52) e substância utilizada a cocaína (OR de 2,88; IC 95\%: 1,09 - 7,62) associou-se a razão circunferência cintura/estatura RCEst (Tabela IV).

Tabela IV - Análise não ajustada de associação entre características socioeconômicas, demográficas e substância utilizada com a razão circunferência cintura/estatura (RCEst) de usuários assistidos em um Centro de Atenção Psicossocial em Álcool e Drogas. São Luís, Maranhão, 2018.

\begin{tabular}{lccc}
\hline & \multicolumn{3}{c}{ Razão circunferência cintura/estatura (RCEst) } \\
Variável & OR & IC 95\% & p-valor \\
\hline Idade (anos) & 1 & & \\
20 a 39 anos & 4,13 & $(1,54-11,05)$ & 0,005 \\
40 a 59 anos & & & \\
Estado civil & 1 & - & 1 \\
$\quad$ Sem companheira & 2,30 & $(0,81-6,52)$ & 0,116 \\
Com companheira & & & \\
Substância utilizada cocaína & 1 & - & 1 \\
Não & 2,88 & $(1,09-7,62)$ & 0,032 \\
Sim & & & \\
\hline
\end{tabular}

RCEst: razão cintura/estatura; OR: odds ratio; IC: intervalo de confiança

Por fim, a Tabela $V$ mostra a análise ajustada, na qual permaneceram associados à RCEst, como fator de risco, a idade entre 40 e 59 anos (OR = 4,40; IC 95\%: 1,52-12,75) e substância utilizada a cocaína (OR = 3,27; IC 95\%: $1,15-9,27)$. 
Tabela V - Análise ajustada de associação entre características socioeconômicas, demográficas e substância utilizada com a razão circunferência cintura/estatura (RCEst) de usuários assistidos em um Centro de Atenção Psicossocial em Álcool e Drogas. São Luís, Maranhão, 2018.

\begin{tabular}{lccc}
\hline & \multicolumn{2}{c}{ Razão circunferência cintura/estatura (RCEst) } \\
Variável & OR & IC 95\%) & p-valor \\
\hline Idade (anos) & 1 & - & 1 \\
$\quad 20$ a 39 anos & 4,40 & $(1,52-12,75)$ & $\mathbf{0 , 0 0 6}$ \\
40 a 59 anos & & - & 1 \\
Estado civil & 1 & $(0,44-4,34)$ & 0,568 \\
$\quad$ Sem companheira & 1,39 & & 1 \\
$\quad$ Com companheira & & - & $\mathbf{0 , 0 2 5}$ \\
Substância utilizada cocaína & 1 & $(1,15-9,27)$ & \\
$\quad$ Não & 3,27 & & \\
Sim & & & \\
\hline
\end{tabular}

RCEst: razão cintura/estatura; OR: odds ratio; IC: intervalo de confiança

\section{DISCUSSÃO}

No atual estudo, a idade e o consumo de cocaína apresentaram, na análise não ajustada e ajustada, associação significativa com a RCEst. Ressalta-se que a avaliação de fatores de risco para DCV, pelo parâmetro RCEst, em dependentes químicos, é escassa na literatura.

A RCEst é considerada um parâmetro frequentemente empregado como indicador prático e simples de detectar o risco cardiovascular ${ }^{(15)}$, sendo um importante prognóstico em diferentes faixas etárias ${ }^{(13)}$. A sua utilização contribui de forma benéfica para a saúde pública ${ }^{(16)}$, agindo como método de triagem rápido, de fácil aplicabilidade e percepção, fornecendo resultados relevantes e de confiabilidade ${ }^{(17)}$.

As DCV estão entre as principais causas de óbitos no mundo, levando a consideráveis gastos sociais e econômicos ${ }^{(18)}$. No Brasil, os custos com essas doenças foram de R $\$ 56,2$ bilhões em $2015^{(19)}$. Entre 2008 e 2014 , $6,7 / 1.000$ homens adultos foram internados decorrente de hipertensão arterial sistêmica ${ }^{(20)}$.

Dentre as comorbidades acometidas aos usuários de álcool e outras drogas, destaca-se a hipertensão arterial, favorecendo o surgimento de $\mathrm{DCV}^{(21)}$. A cocaína está relacionada, principalmente, a queixas cardiovasculares, manifestadas através de infarto, hipertensão, taquicardia, dor torácica e acidentes vasculares cerebrais ${ }^{(9)}$. No Brasil, as doenças do aparelho circulatório são a primeira causa de óbitos, com quase $28 \%$ das mortalidades, sendo $26 \%$ do sexo masculino(22).

Com objetivo de ter um olhar mais direcionado à saúde do homem, o Ministério da Saúde publicou a Política Nacional de Atenção Integral à Saúde do Homem (PNAISH), em conjunto com a Política Nacional de Atenção Básica, que agem na promoção de saúde voltado ao sexo masculino, pois são mais suscetíveis a morbimortalidade e resistentes a procurar pelos serviços de saúde, resultando em morbidades, delonga no atendimento e elevado custo à população ${ }^{(23)}$. Torna-se necessário, no entanto, que a PNAISH promova ações direcionadas aos usuários de drogas, tendo em vista que o número de dependentes químicos está cada vez maior, tornando-os suscetíveis às DCV e alterações mentais, sendo um problema de saúde pública.

Nesse contexto, a Política Nacional de Saúde Mental adota estratégias e diretrizes assistenciais a pacientes e familiares que sofrem com transtornos psíquicos, incluindo os causados por dependência química. No âmbito do SUS, são ofertados atendimentos na Rede de Atenção Psicossocial (RAPS), promovendo a inserção nas redes de serviços em diferentes complexidades ${ }^{(24)}$.

No presente estudo, a maioria dos dependentes químicos eram adultos jovens, viviam sem companheira e tinham ensino fundamental completo ou incompleto. Sendo evidenciado em diversos estudos resultados semelhantes ${ }^{(25-27)}$.

Em pesquisa realizada em uma Comunidade Terapêutica no município de Frederico Westphalen, Rio Grande do Sul, com 14 dependentes químicos do sexo masculino, $57 \%$ apresentaram idade de 20 a 39 anos e, com relação ao estado civil, $57,1 \%$ eram solteiros ${ }^{(25)}$.

O uso da droga é cada vez mais precoce ${ }^{(28)}$. Segundo o relatório da Organização das Nações Unidas (ONU), o consumo é maior entre os homens ${ }^{(3)}$. $O$ fato da maioria dos usuários serem solteiros afirma-se pela influência da 
inversão de valores que surge ao enfrentarem as circunstâncias da droga, em virtude dos dependentes químicos priorizarem o consumo da substância ${ }^{(29)}$.

O baixo grau de instrução dos usuários do presente estudo se assemelha a pesquisa desenvolvida no estado do Rio Grande do Sul, com 1.469 prontuários de dependentes químicos atendidos em um ambulatório, havendo $42,7 \%$ dos pacientes com ensino fundamental incompleto ${ }^{(30)}$.

A baixa escolaridade pode ser justificada ao se considerar o déficit de aprendizado resultante do uso de drogas, ocorrido de forma precoce, em que as substâncias agem no SNC causando prejuízos cognitivos referentes ao raciocínio ${ }^{(30)}$.

Quanto à atividade física, os dependentes químicos do presente estudo costumavam fazer algum tipo de atividade física. Resultado semelhante foi encontrado na pesquisa que tinha como objetivo analisar os efeitos da atividade física no tratamento de dependentes químicos. Foram entrevistados 30 homens, com idade de 18 a 35 anos, que eram assistidos em comunidade terapêutica no interior de São Paulo, no qual observou-se que $30 \%$ dos usuários faziam caminhadas, jogos e alongamentos ${ }^{(31)}$.

Os dependentes químicos em tratamento passam por várias intervenções, entre elas as oficinas terapêuticas, em que a atividade física é requerida pelos Relatórios das Conferências Nacionais em Saúde Mental. Dessa forma, o CAPS necessita da inserção do Educador Físico em sua equipe multiprofissional, sendo indispensável para as atividades ofertadas ${ }^{(32)}$. Além disso, o exercício físico proporcionado pelo CAPS-AD auxilia no tratamento, favorecendo a reinserção social e o desenvolvimento cognitivo, emocional e comportamental desses usuários ${ }^{(33)}$.

A maioria dos usuários do presente estudo eram dependentes de álcool e outras drogas, resultado semelhante ao encontrado em pesquisa transversal que analisou a evasão no tratamento ambulatorial de dependentes químicos no Rio Grande do Sul, de coleta secundária, realizada nos anos de 2012 a 2014 e composto por 593 prontuários de dependentes, evidenciando que o maior percentual dos dependentes químicos procuravam atendimento por fazerem uso de múltiplas drogas ${ }^{(34)}$.

Em uma revisão sistemática, mostrou-se a associação dos usuários de crack ao consumo de maconha, álcool, heroína e cocaína. Esses usuários, normalmente, são poliusuários, ou seja, iniciaram com álcool, tabaco e maconha, ou por um consumo simultâneo dessas substâncias ${ }^{(35)}$.

A duração de tratamento dos participantes do atual estudo ocorreu com maior ênfase de 1 a 6 meses. Ao verificar os dados de uma pesquisa que avaliou as mudanças percebidas pelos usuários ativos que frequentavam o Centro há mais de seis meses, $31,6 \%$ relataram frequentar o CAPS-AD por um período de 1 a 2 anos ${ }^{(36)}$. Portanto, percebe-se o abandono ao tratamento entre os dependentes químicos ${ }^{(37)}$.

Este estudo mostra que o início do consumo de droga ocorreu ainda na adolescência. Semelhante ao achado em pesquisa realizada com usuários de crack que foram avaliados em uma Unidade de Adição, na cidade de Porto Alegre, que encontrou que a média de idade de início do uso das substâncias foi de 21,5 $\pm 6,1$ anos ${ }^{(38)}$.

O Plano Emergencial de Ampliação do Acesso ao Tratamento e Prevenção em Álcool e outras Drogas (PEAD) de 2009-2011 orienta promoção da saúde e prevenção voltada ao consumo de drogas ilícitas, que vem crescendo entre crianças e adolescentes. Nessa fase, os jovens têm dificuldade em aceitar orientações dos seus pais, buscando o controle da própria vida e, consequentemente, se afastando da família. A facilidade de acesso e a comercialização de crack favorece a lei da oferta e procura ${ }^{(39)}$.

Das substâncias que os pacientes já fizeram uso, o crack prevaleceu em relação às demais drogas na presente investigação. Já uma pesquisa realizada no CAPS-AD de Curitiba mostrou que o álcool prevaleceu com $71,2 \%{ }^{(27)}$.

Identificaram-se, no presente estudo, dependentes químicos eutróficos e com risco de DCV. Resultado diferente foi encontrado em estudo que teve por objetivo identificar o estado nutricional de usuários adultos, do sexo masculino, atendidos em um ambulatório para dependentes químicos no Sul do Brasil, no qual $88 \%$ estavam com excesso de peso. E dados semelhantes por apresentar risco elevado para DCV de acordo com a $\mathrm{CC}^{(26)}$.

Uma pesquisa realizada com dependentes químicos nas primeiras 24 horas de internação obteve um maior percentual de baixo peso ${ }^{(40)}$. Essas divergências podem ter acontecido em decorrência da ausência de um protocolo no CAPS-AD a ser seguido quanto ao período de realização da avaliação antropométrica.

Os indicadores de risco cardiovascular utilizados no presente estudo (IC, a RCQ, a CC e a RCEst) mostraram risco de desenvolvimento de DCV. A literatura mostra que os indicadores antropométricos expressam melhor o risco cardiovascular, sendo a RCEst um bom indicador para indivíduos obesos ${ }^{(13)}$.

O estudo apresentou limitações quanto à coleta de dados devido a informações inexistentes nos registros, duplicidade de dados e divergências dos prontuários. Além disso, por explanar uma temática na qual são poucos os estudos referidos. 
Um dos pontos fortes do presente estudo é o fato de apresentar a associação, ainda pouco estudada, entre o indicador RCEst e o consumo de droga, colaborando para o fomento do assunto na literatura. Por meio dos resultados, observa-se que os indicadores antropométricos podem ser utilizados para verificar o risco de DCV em dependentes químicos. São considerados métodos não invasivos e de baixo custo, possibilitando um diagnóstico rápido e intervenções imediatas, que poderão contribuir com a prevenção de doenças, impactando na promoção da saúde e minimizando os gastos públicos.

\section{CONCLUSÃO}

O estudo identificou dependentes químicos adultos jovens, eutróficos, com risco de doenças cardiovasculares e associação significativa da relação cintura/estatura com a idade e a utilização de cocaína.

\section{CONFLITOS DE INTERESSE}

Não há conflitos de interesses.

\section{CONTRIBUIÇÕES}

Kamylla Karolynne Bezerra Pontes e Eliziane Gomes da Costa Moura da Silva contribuíram com a elaboração e delineamento do estudo. Ester Barbosa Soares, Adrielle Zagmignan, Izabela Correa Costa e Virgínia Nunes Lima contribuíram com a redação e/ou revisão do manuscrito. Alexsandro Ferreira dos Santos contribuiu com a aquisição, análise e interpretação dos dados. Janaína Maiana Abreu Barbosa contribuiu com a elaboração e delineamento do estudo, a aquisição, análise e interpretação dos dados, e a redação e/ou revisão do manuscrito.

\section{REFERÊNCIAS}

1. Longo MAT. A dependência de substâncias psicoativas na perspectiva da comunidade terapêutica. UNOPAR Cient Cienc Biol Saude. 2015;17(4):286-91.

2. Ferreira ACZ, Capistrano FC, Souza EB, Borba LO, Kalinke LP, Maftum MA. Motivações de dependentes químicos para o tratamento: percepção de familiares. Rev Bras Enferm. 2015;68(3):474-81.

3. United Nations Office on Drugs and Crime. World Drug Report 2016. Vienna: United Nation Publication; 2016.

4. Laranjeira R, Madruga CS, Ilana Pinsky I, Caetano R, Mitsuhiro SS, Castello G. II Levantamento Nacional de Álcool e Drogas (LENAD) - 2012. São Paulo: Instituto Nacional de Ciência e Tecnologia para Políticas Públicas de Álcoole Outras Drogas (INPAD), UNIFESP; 2014.

5. Brasil. Ministério da Saúde. Portaria de Consolidação no 3, 28 de setembro de 2017. Consolidação das normas sobre as redes do Sistema Único de Saúde. Diário Oficial da União; Brasília, 28 de setembro de 2017.

6. Ministério da Saúde (BR). Secretaria de Atenção à Saúde. Departamento de Ações Programáticas Estratégicas. Guia estratégico para o cuidado de pessoas com necessidades relacionadas ao consumo de álcool e outras drogas: Guia AD. Brasília: Ministério da Saúde; 2015.

7. Chaim CH, Bandeira KBP, Andrade AG. Fisiopatologia da dependência química. Rev Med. 2015;94(4):256-62.

8. Oliveira ALM, Morais BP, Carvalho EBS, Jorge FC Junior, Gouvêa GF, Resende IM, et al. A relação entre a intoxicação por cocaína e o infarto agudo do miocárdio. Rev Cad Med. 2019;2(2):101-12.

9. Smer A, Mahfood HT, Alla V. Cocaine-induced isolated right ventricular infarction. The Am J Emerg Med. 2015;33(7):989.

10. Bartolucci J, Nazzal C., Verdugo FJ, Prieto JC, Sepúlveda P, Corbalán R. Características, manejo y evolución intrahospitalaria de usuarios de drogas ilícitas con infarto agudo del miocardio. Rev Méd Chile. 2016;144(1):39-46.

11. Organização Mundial de Saúde. Obesity: preventing and managing the global epidemic. Report of a WHO consultation, Geneva, 3-5 Jun 1997. Geneva: World Health Organization; 1998. 
12. Associação Brasileira para o Estudo da Obesidade e da Síndrome Metabólica. Diretrizes brasileiras de obesidade 2016/ ABESO - Associação Brasileira para o Estudo da Obesidade e da Síndrome Metabólica. $3^{a}$ ed. Itapevi: AC Farmacêutica; 2016.

13. Haun DR, Pitanga FJG, Lessa I. Razão cintura/estatura comparado a outros indicadores antropométricos de obesidade como preditor de risco coronariano elevado. Rev Assoc Med Bras. 2009;55(6):705-11.

14. Queiróga MB. Utilização de medidas antropométricas para a determinação da distribuição de gordura corporal. Rev Bras Ativ Fís Saúde. 1998;3(1):37-47.

15. Carvalho CA, Fonseca PCA, Barbosa JB, Machado SP, Santos AM, Silva AAM. Associação entre fatores de risco cardiovascular e indicadores antropométricos de obesidade em universitários de São Luís, Maranhão, Brasil. Ciênc Saúde Colet. 2015;20(2):479-90.

16. Diniz KO. Índices antropométricos como preditores de hipertensão arterial em idosos residentes em uma cidade de pequeno porte [dissertação]. São Cristóvão: Universidade Federal de Sergipe; 2015.

17. Oliveira CC, Costa ED, Roriz AKC, Ramos LB, Gomes M Neto. Preditores de síndrome metabólica em idosos: uma revisão. Int J Cardiovasc Sci. 2017;30(4):343-53.

18. Lunkes LC, Murgas LDS, Dorneles EMS, Barcellos CM, Rocha M, Machado GJ. Fatores socioeconômicos relacionados às doenças cardiovasculares: uma revisão. Hygeia. 2018;14(28):50-61.

19. Stevens B, Pezzullo L, Verdian L, Tomlinson J, George A, Bacal F. Os custos das doenças cardíacas no Brasil. Arq Bras Cardiol. 2018;111(1):29-36.

20. Dantas RC, Roncalli AG, Dantas DC. Internações por hipertensão arterial essencial em adultos no Brasil [Internet]. 2015 [acesso em 2019 Mar 20]. Disponível em: http://www.cofen.gov.br/anais-do-18o-cbcenf-jatem-numero-isbn_37372.html

21. Scala LCN. Emergências hipertensivas e uso de drogas ilícitas. Rev Bras Hipertens. 2014;21(4):194-202.

22. Ministério do Planejamento Desenvolvimento e Gestão (BR). Instituto Brasileiro de Geografia e Estatística. Brasil em números. Bras Núm. 2018;26(1):1-512.

23. Coelho EBS, Schwarz E, Bolsoni CC, Conceição TB. Política nacional de atenção integral a saúde do homem [Internet]. Florianópolis: Universidade Federal de Santa Catarina; 2018 [acesso em 2019 Mar 05]. Disponível em: http://portalarquivos2.saude.gov.br/images/pdf/2018/novembro/07/livroPol--ticas-2018.pdf

24. Ministério da Saúde (BR). Secretaria de Atenção à Saúde. Nota Técnica n 11/2019. Esclarecimentos sobre as mudanças na Política Nacional de Saúde Mental e nas Diretrizes da Política Nacional sobre Drogas. Brasília: Ministério da Saúde; 2019.

25. Sirtuli JF, Deon RG, Volkweis DSH, Benetti F. Hábitos alimentares e estado nutricional de dependentes químicos e alcoolistas em uma comunidade terapêutica. Perspectiva, Erechim. 2015;39(145):121-30.

26. Ferreira IB, Paiva CB, Narvaez JCM, Bosa VL. Estado nutricional e hábitos alimentares de dependentes químicos em tratamento ambulatorial. J Bras Psiquiatr. 2015;64(2):146-53.

27. Oliveira VC, Capistrano FC, Ferreira ACZ, Kalinke LP, Felix JVC, Maftum MA. Perfil sociodemográfico e clínico de pessoas atendidas em um CAPS AD do Sul do Brasil. Rev Baiana Enferm. 2017;31(1):1-12.

28. Gusmão PP, Fernandes RFD, Rezende RC, Bonfim RS, Porto YV, Fernandes LC, et al. Perfil epidemiológico de uma população de usuários de drogas de Anápolis, Goiás. Rev Educ Saúde. 2017;5(1):28-37.

29. Freitas EAM, Luís MAV. Perception of students about alcohol consumption and illicit drugs. Acta Paul Enferm. 2015;28(5):408-14.

30. Mascarenhas MA, Santos P, Alves M, Rosa CB, Wilhelms N Junior, Mascarenhas R. Characterization of users of psychoactive substances at the clinic for addictive disorder with emphasis on chemical dependence. Rev Baiana Saúde Pública. 2014;38(4):837-53.

31. Mialick ES, Fracasso L, Sahd SMPV. A importância da prática da atividade física como auxílio no processo de tratamento para a dependência química em pessoas de 18 a 35 anos. Belo Horizonte: Cooperativa do Fitness; 2010 [Internet] [acesso em 05 Mar 2019]. Disponível em: http://www.cdof.com.br/A\%20 
import\%E2ncia\%20da\%20pr\%E1tica\%20de\%20atividade\%20f\%EDsica\%20como\%20aux\%EDlio\%20no\%20 processo $\% 20$ de $\% 20$ tratamento\%20para\%20a\%20depend\%EAncia\%20qu\%EDmica\%20em\%20pessoas\%20 de $\% 2018 \% 20 a \% 2035 \% 20$ anos.pdf

32. Ministério da Saúde (BR). Saúde mental no SUS: os centros de atenção psicossocial. Brasília: Ministério da Saúde; 2004.

33. Silva PPC, Santos ARM, Santos PJC, Rodrigues EAPC, Freitas CMSM. Práticas corporais no Centro de Atenção Psicossocial de Álcool e Drogas: a percepção dos usuários. Rev Bras Ciênc Esporte. 2019;41(1):39.

34. Fernandes SS, Marcos CB, Kaszubowski E, Goulart LS. Evasão do tratamento da dependência de drogas: prevalência e fatores associados identificados a partir de um trabalho de Busca Ativa. Cad Saúde Colet. 2017;2(2):131-7.

35. Teixeira MB, Engstrom EM, Ribeiro JM. Revisão sistemática da literatura sobre crack: análise do seu uso prejudicial nas dimensões individual e contextual. Saúde Debate. 2017;41(112):311-30.

36. Boska GA, Claro HG, Pinho PH, Oliveira MAF. Mudanças percebidas por usuários de centros de atenção psicossocial em álcool e outras drogas. Rev Enferm UFPE on line. 2018;12(2):439-46.

37. Fernandes SS, Marcos CB, Kaszubowski E, Goulart LS. Evasão do tratamento da dependência de drogas: prevalência e fatores associados identificados a partir de um trabalho de Busca Ativa. Cad Saúde Colet. 2017;25(2):131-7.

38. Willhelm FF, Escobar M, Perry IDS. Alterações na composição corporal e em parâmetros antropométricos de dependentes de crack internados em unidade de adição. J Bras Psiquiatr. 2013;62(3):183-90.

39. Moreira MR, Fernandes FMB, Ribeiro JM, Franco NTL. A review of Brazilian scientific output on crack contributions to the political agenda. Cien Saúde Colet. 2015;20(4):1047-62.

40. Balbinot AD, Alves GSL, Amaral AFA Junior, Araújo RB. Perfil antropométrico de dependentes de crack hospitalizados para desintoxicação. Rev HCPA. 2011;31(3):311-7.

\section{Endereço do primeiro autor:}

Kamylla Karolynne Bezerra Pontes

Universidade Ceuma - UNICEUMA

Campus Renascença

Rua Josué Montello, 1

Bairro: Renascença II

CEP: 65075-120 - São Luís - MA - Brasil

E-mail: kamyllakarol@gmail.com

Como citar: Pontes KKB, Soares EB, Santos AF, Zagmignan A, Silva EGCM, Costa IC, et al. Risco cardiovascular de usuários de um centro de atenção psicossocial em álcool e drogas. Rev Bras Promoç Saúde. 2019;32:8874. 